УДК 665.6:622.621.64
Анализ критериев эффективности работы сепараторов
для промысловой подготовки углеводородного сырья

\author{
В. В. Пивень, В. С. Щелконогов* \\ Тюменский индустриальный университет, г. Тюмень, Россия \\ *e-mail: shchelkonogov.slava@mail.ru
}

Аннотация. Оценка качества сепарации углеводородного сырья при его промысловой подготовке необходима для определения эффективности всего технологического процесса подготовки. Отсутствие комплексных критериев оценки качества сепарации для многостадийных процессов не позволяет получить однозначные решения при обосновании технологических схем и оборудования для сепарации. Проанализирована типичная технологическая схема сепарации углеводородного сырья. Проведен анализ имеющихся критериев разделительных процессов. Для комплексной оценки сепарирующих процессов углеводородного сырья предложено использовать критерий, позволяющий проводить расчет эффективности процесса с учетом возможного получения промежуточных фракций и их качественного состава.

Ключевые слова: промысловая подготовка нефти и газа; газовый сепаратор; критерии эффективности сепарации

\title{
Analysis of the criteria of efficiency of work of separators for the field of hydrocarbon raw
}

\section{Valeriy V. Piven, Vladislav S. Shchelkonogov}

Industrial University of Tyumen, Tyumen, Russia *e-mail: shchelkonogov.slava@mail.ru

Abstract. Assessment of the quality of separation of hydrocarbon raw during its field preparation is necessary to determine the efficiency of the entire technological process of preparation. The absence of complex criteria for assessing the quality of separation for multi-stage processes does not allow one to obtain unambiguous solutions in justifying the process flow diagrams and equipment for separation. We have analyzed the typical technological scheme of separation of hydrocarbon raw materials and existing criteria for separation processes. It is proposed to use the criterion for a comprehensive assessment of the separation processes of hydrocarbon raw materials, allowing calculating the efficiency of the process, taking into account the possible production of intermediate fractions and their qualitative composition

Key words: field preparation of oil and gas; gas separator; separation efficiency criteria

\section{Введение}

При разделении скважинной продукции на жидкую и газовую фазы в зависимости от исходного состояния пластовой смеси применяют нефтегазовые или газовые сепараторы [1]. Оценка качества выполнения технологического процесса 
должна производиться по целому ряду критериев. Одним из таких критериев является технологическая эффективность процесса сепарирования скважинной продукции [2].

Без оценки эффективности данного процесса невозможны оптимизация технологических процессов и автоматизация процесса управления сепарацией.

Помимо промысловой подготовки нефти и газа сепараторы являются обязательным элементом технологической цепочки в процессе переработки газового конденсата [3, 4].

В газонефтяных сепараторах газожидкостная смесь имеет небольшое содержание газа, а в газовых сепараторах обрабатывается газожидкостная смесь с относительно небольшим содержанием жидкой фазы. При всем многообразии технологических схем сепарации, применяемых способов сепарации, исходного состояния пластовой смеси задачей сепарирования является получение как минимум двух готовых фаз с максимальным выходом жидкой или газовой фазы при минимальных остатках неизвлеченного продукта [5-7].

Решение оптимизационной задачи с поиском решения, когда, по крайней мере, две функции должны иметь глобальный максимум, а еще две функции одновременно такой же минимум, почти невозможно. К тому же на целесообразность применения конкретной схемы сепарации должны накладываться и стоимостные ограничения.

В связи с этим обоснование комплексных критериев технологической эффективности процесса сепарации является актуальной научной проблемой.

\section{Объект и методы исследования}

При промысловой подготовке нефти и газа необходимо довести скважинную продукцию до товарных кондиций согласно техническим требованиям по ГОСТ Р 51858-2002 ${ }^{1}$ на нефть (табл. 1) и ОСТ 51.40-93 ${ }^{2}$ на газ (табл. 1 и 2). Стандартами устанавливаются только требования к конечному продукту, прошедшему сепарацию. Качество выполнения технологического процесса отдельными машинами или отдельными секциями в составе машины не регламентируется. Поэтому на стадии проектирования при эксплуатации оборудования для подготовки нефти и газа для оптимизации технологического процесса необходимо применение критериев эффективности разделительных процессов.

таблица 1

Технические условия (ГОСТ Р 51858-2002) на группы качества подготовки товарной нефти

\begin{tabular}{|l|c|c|c|}
\hline \multirow{2}{*}{ Показатель } & \multicolumn{3}{|c|}{$\begin{array}{c}\text { Нормы показателя } \\
\text { для группы качества }\end{array}$} \\
\cline { 2 - 4 } & 1 & 2 & 3 \\
\hline Массовая доля воды, \%, не более & 0,5 & 0,5 & 1,0 \\
\hline Концентрация хлористых солей, $\Gamma \mathrm{M}^{3}$ (мг/дм ${ }^{3}$, мг/л), не более & 100 & 300 & 900 \\
\hline Массовая доля механических примесей, \%, не более & \multicolumn{3}{|c|}{0,05} \\
\hline $\begin{array}{l}\text { Давление насыщенных паров при температуре } 37,8^{0} \mathrm{C} \\
\text { в бомбе Рейда, кПа (мм рт. ст.), не более }\end{array}$ & $\begin{array}{c}66,7 \text { (500 мм рт. ст.) } \\
\text { при температуре } 37,8^{0} \mathrm{C}\end{array}$ \\
\hline Содержание хлорорганических соединений, млн ${ }^{-1},(\mathrm{ppm})$ & $\begin{array}{c}\text { Не нормируется } \\
\text { Определение обязательно }\end{array}$ \\
\hline
\end{tabular}

${ }^{1}$ ГОСТ Р 51858-2002. Нефть. Общие технические условия. - Введ. 2002-07-01. - М.: Стандартинформ, 2006. - 12 с.

${ }^{2}$ OCT 51.40-93 Газы горючие природные, поставляемые и транспортируемые по магистральным газопроводам. Технические условия. - Введ. 1993-10-01. - М., 1993. - 8 с. 
В промысловой практике выбор системы сепарации скважинной продукции зависит от ее реологических, физических свойств, а также от диспергирования и коалесценции, которые в свою очередь определяются условиями добычи и технологией подготовки скважинной продукции. При этом данные условия меняются в зависимости от стадии разработки нефтегазового месторождения $[8,9]$.

Таблица 2

Требования к качеству газа по ОСТ 51.40-93

\begin{tabular}{|c|c|c|c|c|}
\hline \multirow{3}{*}{ Параметр } & \multicolumn{4}{|c|}{ Значения для климатических районов } \\
\hline & \multicolumn{2}{|c|}{ умеренного } & \multicolumn{2}{|c|}{ холодного } \\
\hline & $\begin{array}{l}\text { с } 01.05 \\
\text { по } 30.09\end{array}$ & $\begin{array}{c}\text { с } 01.10 \\
\text { по } \\
30.04\end{array}$ & $\begin{array}{l}\text { с } 01.05 \\
\text { по } 30.09\end{array}$ & $\begin{array}{c}\text { с } 01.10 \\
\text { по } 30.04\end{array}$ \\
\hline Точка росы по влаге, ${ }^{0} \mathrm{C}$, не выше & -3 & -5 & -10 & -20 \\
\hline Точка росы по углеводородам, ${ }^{0} \mathrm{C}$, не выше & 0 & 0 & -5 & -10 \\
\hline Масса сероводорода, $\Gamma / \mathrm{m}^{3}$, не более & \multicolumn{4}{|c|}{0,007} \\
\hline Масса меркаптановой серы, г/м³, не более & \multicolumn{4}{|c|}{0,016} \\
\hline Объемная доля кислорода, \%, не более & \multicolumn{2}{|c|}{0,5} & \multicolumn{2}{|c|}{1,0} \\
\hline $\begin{array}{l}\text { Теплота сгорания низшая МДж/м } \\
\text { при } 20{ }^{\circ} \mathrm{C} \text { и } 101,325 \text { кПа, не менее }\end{array}$ & \multicolumn{4}{|c|}{32,5} \\
\hline Температура газа, ${ }^{0} \mathrm{C}$ & \multicolumn{4}{|c|}{$\begin{array}{c}\text { На входе и в самом газопроводе } \\
\text { устанавливается проектом }\end{array}$} \\
\hline $\begin{array}{l}\text { Масса механических примесей } \\
\text { и труднолетучих жидкостей }\end{array}$ & \multicolumn{4}{|c|}{$\begin{array}{c}\text { Условия оговариваются в соглашениях на } \\
\text { поставку газа с ПХГ, ГПЗ и промыслов }\end{array}$} \\
\hline
\end{tabular}

Технологический процесс в нефтегазовых сепараторах (рисунок) состоит из подачи исходной смеси по патрубку (1) в раздаточный коллектор (2) [10]. Регулятор давления (3) обеспечивает снижение давления по сравнению с исходным. При снижении давления из смеси выделяется растворенный газ.

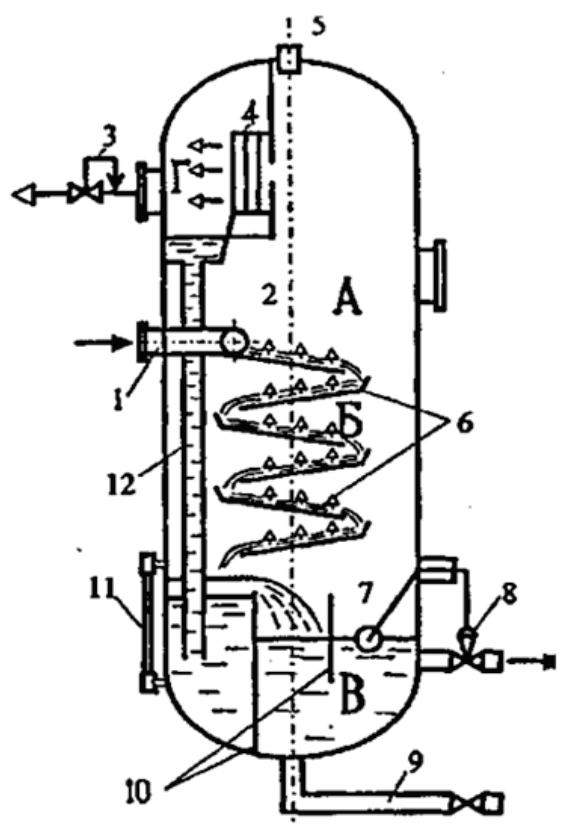

\footnotetext{
Рисунок. Схема вертикального сепаратора с цилиндрическим корпусом: A-основная сепарационная секция; Б- осадительная секция; В - секция сбора нефти;

Г- секция каплеотделения; 1 - патрубок ввода зазожидкостной смеси; 2 - раздаточный коллектор со щелевым выходом; 3 - регулятор давления «до себя» на линии отвода газа; 4 - жалюзийный каплеуловитель;

5 - предохранительный клапан; 6 - наклонные полки; 7 - поплавок; 8 - регулятор уровня на линии отвода нести; 9 - линия сброса шлама; 10 - перегородки;

11 - уровнемерное стекло; 12 - дренажная труба
} 
Для более полного выделения растворенного газа необходимо определенное время нахождения смеси в сепараторе. Для этого установлены наклонные полки (6), по которым смесь стекает в нижнюю часть аппарата. За счет этого увеличивается траектория движения смеси.

На заключительном по ходу технологического процесса этапе в верхней части сепаратора полученный газ проходит еще один этап: на жалюзийной каплеуловительной насадке (4) отделяются капли жидкой фракции, увлеченные потоком газа.

Для более эффективного использования рабочего пространства сепаратора необходима оптимальная компоновка рабочих секций сепаратора с учетом их сепарирующих возможностей, производительности и исходного состава сепарируемой смеси. Возможна организация технологического процесса сепарации по разветвленной схеме, когда для повышения удельной производительности исходный обрабатываемый продукт разделяется на несколько потоков [11-14].

При одинаковом исходном составе полученные потоки следует разделять по параллельным схемам. В случае разделения поступающей в сепаратор смеси на разнокачественные потоки их последующую сепарацию необходимо производить на соответствующих рабочих органах. Время обработки и траектория движения таких потоков могут отличаться, что в конечном итоге позволит повысить удельную производительность [15].

\section{Результаты}

Оценка технологической эффективности делительной способности сепараторов производится с помощью безразмерного коэффициента эффективности $\eta$, который равен отношению объема жидкой фазы, осевшей в сепараторе, к количеству жидкой фазы, содержащейся на входе в сепаратор [3].

$$
\eta=\frac{W_{S}}{W_{0}}=\frac{W_{0}-W_{1}}{W_{0}}=1-\frac{W_{1}}{W_{0}}=1-K
$$

где $W_{0}, W_{l}, W_{S}$ - объемные содержания жидкой фазы на входе и выходе сепаратора и доля объема, осевшего в сепараторе; $K$ - коэффициент уноса (характеризует количество жидкой фазы, уносимой с потоком газа).

В работе [16] получены зависимости для определения коэффициента эффективности вертикального сепаратора в зависимости от распределения капель жидкой фазы на входе, выходе и от их размера:

$$
\eta=1-\frac{\exp \left(-3 \sigma^{2}\right)}{\sqrt{2 \pi} \sigma} \int_{0}^{z_{m}} z^{2}\left(1-\frac{\tau z^{2}}{1+0,032 A r_{a v}^{0,5} z^{3 / 2}}\right) \exp \left[-\frac{\ln ^{2}\left(z / z_{1}\right)}{2 \sigma^{2}}\right] d z .
$$

где

$$
\begin{gathered}
z=\frac{R}{R_{a v}}, \\
z_{1}=\frac{R_{1}}{R_{a v}}=\exp \left(-0,5 \sigma^{2}\right), \\
\tau=\frac{t}{t_{d s}}=\frac{z u_{s}}{U D}, \\
A r_{a v}=\frac{8 R_{a v}^{3} \rho_{G} V \rho g}{\mu_{G}^{2}}, \\
z_{m}=\frac{R_{m}}{R_{a v}},
\end{gathered}
$$

где $R$ - радиус капли; $R_{a v}$ - средний радиус капли; $\sigma$ - дисперсия распределения капель по радиусам; $t$ - время нахождения смеси в сепараторе; $t_{d s}$ - время, за 
которое капля радиуса $R$ выпадает из слоя высоты $D ; U$ - средняя скорость потока; $R_{m}$ - минимальный радиус капли.

Коэффициент эффективности работы вертикального сепаратора [4]

$$
\eta=1-\frac{\exp \left(-3 \sigma^{2}\right)}{\sqrt{2 \pi} \sigma} \int_{0}^{z_{m}} z^{2} \exp \left[-\frac{\ln ^{2}\left(z / z_{1}\right)}{2 \sigma^{2}}\right] d z
$$

где $z_{m}=\frac{R_{m}}{\sqrt{9 \mu_{G} U / 2 \Delta \rho g}} \times \sqrt{\frac{U}{u_{s}}} ; U-$ скорость движения потока; $u_{s}-$ скорость осаждения капли.

\section{Обсуждение}

Имеющиеся зависимости для оценки технологической эффективности не позволяют дать однозначный ответ на вопрос о том, что предпочтительнее: получить в сепараторе высокую производительность с большим коэффициентом уноса или невысокую производительность с небольшим коэффициентом уноса [17]. В первом случае с высокой производительностью можно было бы для дополнительного извлечения жидкой фазы из потока газа произвести эту операцию на отдельном небольшом аппарате. В конечном итоге только с учетом экономической оценки всего технологического процесса можно было бы дать окончательное решение.

При разделении исходной смеси на несколько неоднородных потоков и их раздельной сепарации количество получаемых в результате сепарации промежуточных фракций может быть как минимум больше двух. Эти фракции также будут иметь разный состав, в том числе это могут быть конечные фракции, не требующие дальнейшей сепарации.

Для сепарации $n$-компонентной сыпучей смеси на $n$ фракций В. М. Цециновским [18] предложено оценивать технологическую эффективность сепарирования выражением (9).

$$
E_{n}=\sum W_{i}\left(\varphi_{i i}-a_{i}\right) /\left(a_{i i}-a_{i}\right),
$$

где $W_{i}$ - выход $i$-й фракции в долях единицы; $\varphi_{i i}$ - содержание $i$-го компонента в $i$-й фракции или фактическая чистота фракции в долях единицы; $a_{i}-$ содержание $i$-го компонента в исходной смеси в долях единицы; $a_{i i}-$ содержание $i$-го компонента в $i$-й фракции при предельном разделении или предельная чистота фракции в долях единицы.

Слагаемые в выражении (9) представляют собой произведение количественного эффекта $\left(W_{i}\right)$ на качественный эффект. Каждое из слагаемых характеризует частный эффект сепарирования каждой из фракций.

\section{Выводы}

Таким образом, приведенная зависимость позволяет определять технологическую эффективность сепарирования при условии, если известны выходы и чистота полученных фракции, а также чистота фракций при предельном сепарировании.

Выражение (9) позволяет комплексно учитывать долю жидкой фазы, осевшей в сепараторе, а также унос жидкой фазы с потоком газа. При обосновании конструкций сепараторов с развитой технологической схемой и последовательнопараллельным соединением рабочих органов данную зависимость можно использовать как основную для совершенствования критериев оценки технологической эффективности сепарации и экономико-математического моделирования всего процесса сепарации. 


\section{Библиографический список}

1. Марченко В. В., Музипов Х. Н. К вопросу подготовки нефти // Вестник магистратуры. - 2017. - № 68. - С. 70-74.

2. Пивень В. В., Щелконогов В. С. Анализ конструкций и тенденции развития газовых сепараторов // Состояние, тенденции и проблемы развития нефтегазового потенциала Западной Сибири. - Тюмень: ТИУ, 2018. - 287 с.

3. Технологические основы и моделирование процессов промысловой подготовки нефти и газа: учеб. пособие / Н. В. Ушева [и др.]; Томский политехнический университет. 2-е изд. - Томск: Изд-во Томского политехнического ун-та, 2013. - 128 с.

4. Молчанов Г. В., Молчанов А. Г. Машины и оборудование для добычи нефти и газа: учеб. для вузов. - М.: Недра, 1984. - 464 с.

5. Мильштейн Л. М., Бойко С. И., Запорожец Е. П. Нефтегазопромысловая сепарационная техника: справ. пособие / Под ред. Л. М. Мильштейна. - М.: Недра, 1992. - 236 с.

6. Белевицкий А. М. Проектирование газоочистительных сооружений. - Л.: Химия. 1990. $-288 \mathrm{c}$.

7. 7. Song X. L. Research on Separation Efficiency for Oil/Gas Separator of Submersible Electrical Pump // Applied Mechanics and Materials. - 2013. - Vol. 295-298. - P. 3261-3264. DOI: 10.4028/www.scientific.net/AMM.295-298.3261

8. Трубопроводный транспорт нефти и газа: учеб. для вузов / Р. А. Алиев [и др.]. 2-е изд., перераб. и доп. - М.: Недра, 1988. - 368 с.

9. Гриценко А. И., Александров И. А., Галанин И. А. Физические методы переработки и использование газа: учеб. пособие. - М.: Недра, 1981. - 224 с.

10. Сваровская Н. А. Подготовка, транспорт и хранение скважинной продукции: Учебное пособие. - Томск: Изд-во ТПУ, 2004. - 268 с.

11. Arnold K., Steward M. Surface Production Operations. - Houston; Texas: Gulf Publishing Company, 1999. $-584 \mathrm{p}$.

12. Шаймарданов В. Х. Процессы и аппараты технологий сбора и подготовки нефти и газа на промыслах: учеб. пособие / Под ред. В. И. Кудинова. - М. - Ижевск: НИЦ «Регулярная и хаотическая динамика», Институт компьютерных исследований, 2013. -508 с.

13. Gas evolution rates - A critical uncertainty in challenged gas-liquid separations / D. M. Lavenson [et al.] // Journal of Petroleum Science and Engineering. - 2016. - Vol. 147. P. 816-828. DOI: 10.1016/j.petrol.2016.10.005

14. Investigation of the oil-gas separation in a horizontal separator for oil-injected compressor units / J. Feng [et al.] // Proceedings of the Institution of Mechanical Engineers, Part A: Journal of Power and Energy. - 2008. - Vol. 222, Issue 4. - P. 403-412. DOI: 10.1243/09576509JPE550

15. Baltrenas P., Chlebnikovas A. Investigation into a new generation multi-channel cyclone used for removing lignin particulate matter from gas under conditions of an aggressive environment // Process Safety and Environmental Protection. - 2016. - Vol. 99. - P. 107-119. DOI: 10.1016/j.psep.2015.10.014

16. Синайский Э. Г., Лапига Е. Я., Зайцев Ю. В. Сепарация многофазных многокомпонентных систем. - М.: ООО «Недра-Бизнесцентр», 2002. - 621 с.

17. Пивень В. В., Уманская О. Л. Анализ методов оценки эффективности сепарирования [Электронный ресурс] // Современные научные исследования и инновации. - 2013. - № 2. Режим доступа: http://web.snauka.ru/issues/2013/02/22518 (дата обращения: 23.09.2018).

18. Процессы сепарирования на зерноперерабатывающих предприятиях / В. В. Гортинский [и др.] - 2-е изд., перераб. и доп. - М.: Колос, 1980. - 304 с.

\section{Сведения об авторах}

Пивень Валерий Васильевич, д. т. н., профессор кафедры машин и оборудования нефтяной и газовой промышиленности, Тюменский индустриальный университет, 2. Тюмень, e-mail:pivenvv@yandex.ru

Щелконогов Вячеслав Сергеевич, магистрант, Тюменский индустриальный университет, г. Тюмень, e-mail: shchelkonogov.slava@mail.ru

\section{Information about the authors}

Valeriy V. Piven, Doctor of Engineering, Professor at the Department of Machinery and Equipment for the Oil and Gas Industry, Industrial University of Tyumen, e-mail: pivenvv@yandex.ru

Vladislav S. Shchelkonogov, Master's Student, Industrial University of Tyumen, e-mail: shchelkonogov.slava@mail.ru 